\title{
Prognostic significance of low DICER expression regulated by miR-130a in cervical cancer
}

\author{
L He ${ }^{1,2,3}$, H-Y Wang ${ }^{*, 1}$, L Zhang ${ }^{1,2}$, L Huang ${ }^{1}$, J-D Li ${ }^{1,2}$, Y Xiong ${ }^{1,2}$, M-Y Zhang ${ }^{1}$, W-H Jia ${ }^{1}$, J-P Yun ${ }^{1,4}$, R-Z Luo ${ }^{1,4}$ and M Zheng,
}

Dicer is crucial for the maturation of microRNAs (miRNAs) and its dysregulation may contribute to tumor initiation and progression. The study explored the clinical implications of Dicer and its post-transcriptional regulation by microRNAs in cervical cancer. qRT-PCR and immunohistochemistry investigated Dicer mRNA and protein levels in cervical cancer tissues. The relationship between Dicer expression and survival was analyzed. MiRNA target prediction identified miRNAs that might target Dicer. Luciferase reporter and gain- or loss-of-function assays were performed. The results showed that $36.7 \%$ of cervical cancer cases showed low expression of Dicer mRNA and $63.3 \%$ cases showed high expression. At the protein level, $51 \%$ cases showed negative expression and $49 \%$ cases showed positive expression. Dicer mRNA and protein expressions were significantly associated with distant metastasis and recurrence in cervical cancer $(P=0.002$ and $P=0.012$, respectively). Multivariate Cox analysis indicated that low Dicer expression $(P=0.016)$ and tumor stage $(P=0.047)$ were independent predictors. Among the miRNAs predicted to target Dicer, 10 were detected by RT-PCR; their expressions were significantly higher in cervical cancers with lower Dicer expression than in those with higher Dicer expression and were negatively correlated with Dicer expression level $(P<0.05)$. In vitro experiments demonstrated that miR-130a directly targeted Dicer mRNA to enhance migration and invasion in SiHa cells. Finally, survival analysis indicated that higher expression of miR-130a was significantly associated with poor disease-free survival. Taken together, Dicer expression regulated by miR-130a is an important potential prognostic factor in cervical cancer.

Cell Death and Disease (2014) 5, e1205; doi:10.1038/cddis.2014.127; published online 1 May 2014

Subject Category: Cancer

Dicer is a cytoplasmic RNase III enzyme that cleaves the loop of the pri-miRNA and long double-stranded RNA into $\sim 22$ bp double-stranded miRNA and short interfering RNA (siRNA) that target specific messenger RNAs, resulting in gene silencing. Dicer has an important role in the regulation of cell number and in controlling apoptosis. ${ }^{1-3}$ Loss of Dicer in mice disrupts embryonic stem-cell differentiation and is lethal during early development. ${ }^{4}$ Low Dicer expression is associated with worse clinical outcomes in lung cancer, ${ }^{5}$ breast cancer, ${ }^{6}$ and endometrial adenocarcinoma. ${ }^{7}$ Low expression of Dicer and Drosha is associated with ovarian cancer progression and poor clinical outcomes. $^{8}$

MiRNAs are small non-coding RNAs that modulate gene expression, mainly by base pairing to the $3^{\prime}$-untranslated region (UTR) of the target mRNA post-transcriptionally. ${ }^{9}$ They are predicted to control $>60 \%$ of human genes. ${ }^{10}$ They have important roles in development, cellular differentiation, proliferation, cell cycle control and cell death, ${ }^{11}$ and have been implicated in a variety of human diseases, including cancer. ${ }^{11,12}$ For example, miRNA-130a antagonizes the inhibitory effects of GAX on endothelial cell proliferation, migration, and the inhibitory effects of HoxA5 on tube formation in vitro. ${ }^{13}$ MiRNA-130a overexpression was associated with lymph node metastasis and poor prognosis of non-small cell lung cancer (NSCLC). ${ }^{14}$ Higher expression of MiRNA-103/107, which attenuates miRNA biosynthesis by targeting and inhibiting Dicer, causes global miRNA downregulation and acts as a prognostic marker in breast tumors. ${ }^{15}$ Dicer-targeting miRNAs regulate Dicer expression and constitute a negative feedback loop. ${ }^{16}$ There is increasing evidence that the expression of miRNA genes is aberrant in cervical cancer, and a subset of miRNAs is identified that correlate with disease stage and recurrence. ${ }^{17-19}$ Till now, there has been no study on Dicer expression in cervical carcinoma.

\footnotetext{
${ }^{1}$ State Key Laboratory of Oncology in Southern China, Sun Yat-Sen University Cancer Center, 651 Dongfeng Road East, Guangzhou 510060, China; ${ }^{2}$ Department of Gynecology, Sun Yat-Sen University Cancer Center, 651 Dongfeng Road East, Guangzhou 510060, China; ${ }^{3}$ Department of Obstetrics and Gynecology, The First Affiliated Hospital, Sun Yat-sen University, Guangzhou, China and ${ }^{4}$ Department of Pathology, Sun Yat-Sen University Cancer Center, 651 Dongfeng Road East, Guangzhou 510060, China

*Corresponding author: H-Y Wang, State Key Laboratory of Oncology in Southern China, Sun Yat-Sen University Cancer Center, 651 Dongfeng Road East, Room 617, Guangzhou 510060, China. Tel/Fax: + 86208734 3308; Email: wanghyun@mail.sysu.edu.cn

or M Zheng, State Key Laboratory of Oncology in Southern China and Department of Gynecology, Sun Yat-Sen University Cancer Center, 651 Dongfeng Road East, Guangzhou, 510060, China. Tel: + 86 13926162688; Fax: + 8620 32968086; E-mail: zhengmin@sysucc.org.cn

Keywords: Dicer; miR-130; cervical cancer; microRNA; prognostic factor

Abbreviations: siRNA, short interfering RNA; $3^{\prime}$-UTR, $3^{\prime}$-untranslated region; NSCLC, non-small cell lung cancer; ROC curve, receiver operating characteristic curve; IHC, immunohistochemistry; qRT-PCR, quantitative real-time polymerase chain reaction; SCC, squamous cell carcinoma antigen; OS, overall survival; DFS, diseasefree survival; HR, hazard ratio; NC, negative control; BC, blank control; TRBP protein, the TAR-binding protein; WHO classification criteria, World Health Organization classification criteria; RPMI 1640, Roswell Park Memorial Institute 1640; FBS, fetal bovine serum; Ct, threshold cycle; FIGO Stage, Federation of Gynecology and Obstetrics Stage; $\mathrm{Cl}$, Confidence Interval

Received 27.7.13; revised 25.2.14; accepted 28.2.14; Edited by G Melino
} 
In this study, we investigated Dicer expression in a large cohort of primary invasive cervical carcinomas. Using an in silico approach, we identified several conserved miRNAs predicted to target the $3^{\prime}$-UTR of Dicer. Then, we investigated the role of miRNAs in the regulation of Dicer expression and revealed the upstream regulation mechanism. Finally, we explored the function of miR-130a in cervical cancer cell lines.

\section{Results}

Expression of Dicer in cervical carcinoma. We detected Dicer mRNA levels in 90 cervical cancer tissues and 23 adjacent non-cancerous tissues using qRT-PCR. The Dicer mRNA levels in the cervical cancer tissues were not normally distributed $(P=0.013$ by the KolmogorovSmirnov test for normality). A histogram of Dicer expression showed a frequency distribution with two prominent peaks at log2 values from -1.0 to -0.67 and from 0 to 0.33 . A ROC curve was therefore used to identify the cut-off value with the highest potential for discriminating two distinct groups in terms of the log2 ratio of Dicer expression $(P=0.007)$ (Figure 1A). The value was found to be -0.0192 , which was close to its mean values $(0.15)$. Dicer mRNA expression varied among cervical cancer specimens. Low Dicer expression was observed in $36.7 \%$ of samples; high Dicer mRNA expression was observed in $63.3 \%$ of samples. The median ratio of Dicer expression in cancer specimens with low Dicer mRNA expression was 0.578 (range, 0.18-0.99); specimens with high Dicer mRNA expression had a median ratio of 1.47 (range, 0.99-6.03). To determine whether Dicer mRNA levels reflected protein expression, 102 cervical cancers specimens, including samples from the same 90 cases detected by qRT-PCR, were also examined using immunohistochemistry (IHC) (Table 1). Fifty-two cases (51\%) were negative for Dicer expression, whereas 50 cases (49\%) were positive for Dicer. The IHC score agreed with the qRT-PCR results for Dicer (kappa $=0.717$; 95\% confidence interval $(\mathrm{Cl}), 0.704-$ $0.853)$. In the 23 pairs of samples, the relative mRNA expression of Dicer was not significantly different between cervical cancer tissues and the matched adjacent noncancerous tissues ( $P=0.2528$ ) (Figures $1 \mathrm{~B}$ and D). However, IHC analysis showed that Dicer protein expression in the cervical cancer samples was much higher than in the matched adjacent non-cancerous samples $(n=23$, $P<0.05$ ) (Figures $1 \mathrm{C}$ and $\mathrm{D}$ ). The inconsistency between Dicer mRNA and protein level in cervical cancer indicated that a post-transcriptional mechanism is involved in regulating Dicer expression in cervical cancer.

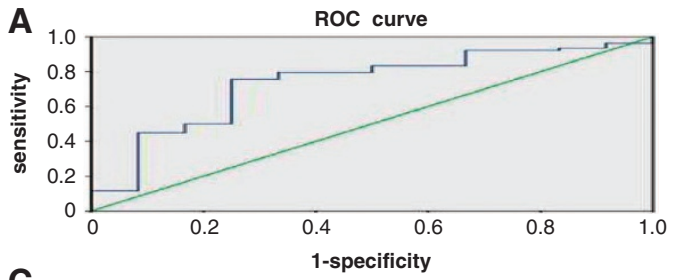

B

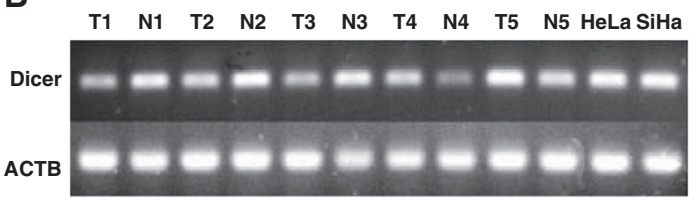

C
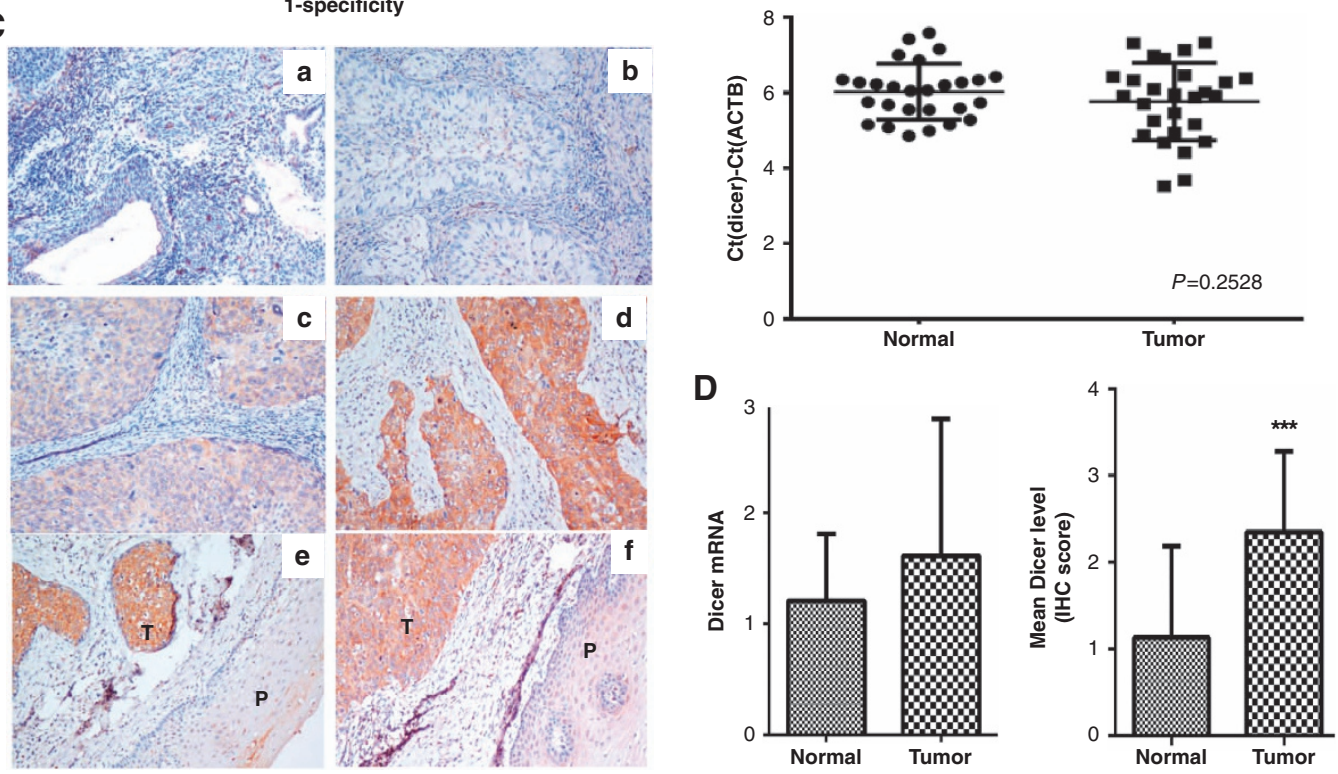

Figure 1 (A) A ROC curve was used to discriminate two distinct groups of Dicer expression. (B) The mRNA expression of Dicer was not significantly different between cervical cancer tissues and matched adjacent non-cancerous tissues. (C) Dicer protein levels in cervical cancer tissues and adjacent non-cancerous tissues. (a) Cervical squamous cell cancer, rated Dicer $(0)(\times 200)$. (b) Cervical adenocarcinoma, rated Dicer $(1)(\times 200)$. (c) Cervical squamous cell cancer, rated Dicer $(2)(\times 200)$. (d) Cervical squamous cell cancer, rated Dicer $(3)(\times 200)$. $(e, f)$ Immunohistochemistry showing high expression of Dicer in cervical squamous cell cancer, and low expression of Dicer in adjacent non-cancerous tissues. $t$, tumor; $p$, adjacent non-cancerous tissues. (D) Dicer protein expression in the cervical cancer samples was much higher than that in the matched adjacent non-cancerous samples 
Table 1 Correlations between Dicer expression and clinicopathological features of patients with cervical carcinoma

\begin{tabular}{|c|c|c|c|c|c|c|}
\hline \multirow[t]{3}{*}{ Characteristics } & \multirow{2}{*}{\multicolumn{3}{|c|}{$\begin{array}{l}\text { IHC data set } \\
(n=102) \\
\text { DICER }\end{array}$}} & \multirow{2}{*}{\multicolumn{3}{|c|}{$\begin{array}{l}\text { qRT-PCR data set } \\
(n=90) \\
\text { DICER }\end{array}$}} \\
\hline & & & & & & \\
\hline & High & Low & $P^{\star}$ & High & Low & $P^{*}$ \\
\hline \multicolumn{7}{|l|}{ Age (years) } \\
\hline$>35$ & 36 & 45 & 0.088 & 43 & 26 & 0.8 \\
\hline$\leqslant 35$ & 14 & 7 & & 14 & 7 & \\
\hline \multicolumn{7}{|l|}{ Tumor size } \\
\hline$\geqslant 4 \mathrm{~cm}$ & 23 & 28 & 0.553 & 26 & 17 & 0.664 \\
\hline$<4 \mathrm{~cm}$ & 27 & 24 & & 31 & 16 & \\
\hline \multicolumn{7}{|l|}{$S C C$} \\
\hline$\geqslant 1.5 \mathrm{ng} / \mathrm{ml}$ & 10 & 18 & 0.125 & 14 & 9 & 0.806 \\
\hline$<1.5 \mathrm{ng} / \mathrm{ml}$ & 40 & 34 & & 43 & 24 & \\
\hline \multicolumn{7}{|l|}{ Histology } \\
\hline Adenocarcinoma & 2 & 4 & 0.678 & 2 & 3 & 1 \\
\hline Squamous & 48 & 48 & & 54 & 31 & \\
\hline \multicolumn{7}{|l|}{ Tumor grade } \\
\hline G1 & 17 & 17 & 1.000 & 20 & 11 & 1 \\
\hline G2/G3 & 33 & 35 & & 37 & 22 & \\
\hline \multicolumn{7}{|l|}{ FIGO stage } \\
\hline$|-| \mid$ & 48 & 41 & 0.015 & 57 & 31 & \\
\hline III-IV & 2 & 11 & & 0 & 2 & \\
\hline \multicolumn{7}{|c|}{ Lymph node metastasis } \\
\hline Yes & 14 & 8 & 0.335 & 13 & 9 & 0.8 \\
\hline No & 36 & 35 & & 44 & 24 & \\
\hline Missing data & 0 & 9 & & & & \\
\hline \multicolumn{7}{|c|}{ Distant metastasis and recurrence } \\
\hline Yes & 7 & 19 & 0.012 & 7 & 14 & 0.002 \\
\hline No & 43 & 33 & & 50 & 19 & \\
\hline
\end{tabular}

Abbreviations: FIGO, International Federation of Gynecology and Obstetrics; SCC, squamous cell carcinoma antigen

$P^{\star}: P$-values were calculated with a two-tailed Fisher's exact test

Low expression of Dicer was associated with clinical stage and recurrence. We then analyzed the relationships between Dicer expression level (mRNA and protein) and the clinical characteristics of 102 patients with invasive cervical carcinoma. Chi-square tests showed that neither Dicer mRNA nor Dicer protein level was significantly associated with age, tumor grade, histology, tumor size, lymph node metastasis or squamous cell carcinoma antigen (SCC) level (Table 1). However, Dicer protein expression was significantly associated with advanced tumor stage $(P=0.015)$. Both mRNA and protein expression levels were significantly associated with recurrence, including distant metastasis and local recurrence ( $P=0.002$ and $P=0.012$, respectively). Kaplan-Meier survival analyses showed that cervical cancer patients with low Dicer mRNA expression had a significantly shorter 5-year disease-free survival (DFS) (57.6\% versus $87.7 \%$, $P=0.001)$ and 5 -year overall survival (OS) $(72.7 \%$ versus $94.7 \%, P=0.0028)$ than those with high Dicer mRNA expression (Figure 2a). Similarly, patients with low Dicer protein expression were significantly associated with shorter 5-year DFS (63.5\% versus $86.0 \%, P=0.013)$ and 5-year OS (75\% versus $92 \%, P=0.0223)$ than patients with high Dicer protein expression (Figure 2b). Furthermore, univariate Cox regression analysis of various parameters with OS showed that low expression of Dicer (protein hazard ratio (HR): 3.418, $P=0.032$; mRNA HR: 5.794, $P=0.008$ ) and clinical stage (HR: 7.761, $P=0.01$ ) were significant predictive factors for poor outcome. Similar results were observed between DFS and Dicer expression. Multivariate Cox regression showed that low expression of Dicer $(P=0.016)$ and tumor stage $(P=0.047)$ remained independent predictors (Table 2$)$.

Low Dicer expression in cervical cancers is inversely correlated with high expression of 10 miRNAs. We reasoned that the reduced expression of Dicer in cervical cancer might be induced by high expression of miRNAs. Using several miRNA target prediction programs, we found 10 miRNAs (hsa-miR-130a, -130b, -148a, -148b, -29a, -29b, $-29 c,-19 b,-301 a$, and $-301 b)$ that were predicted to target the $3^{\prime}$-UTR of Dicer transcripts. The expression levels of these 10 miRNAs in 26 cervical cancer tissues with low Dicer expression and 29 with high Dicer expression were examined by qRT-PCR. The expression levels of the 10 miRNAs were all significantly higher in the low-Dicer-expression cervical cancers compared with the high-Dicer-expression cervical cancers $(P<0.05)$ (Figure 3a). There was a significant inverse correlation between the expressions of Dicer and the 10 miRNAs in cervical cancer, with Pearson correlation coefficients ranging from -0.29 to -0.44 (Figure $3 b$ ). The data suggested that these miRNAs might target Dicer mRNA.

The relative mRNA expression of Dicer and the 10 miRNAs was determined by qRT-PCR on 55 cervical cancer tissues, 23 adjacent non-cancerous tissues and cervical cancer cells. Data revealed that the Dicer mRNA expression in cancer cells was higher than in the cancer tissues and adjacent non-cancerous tissues (3.05-fold and 2.18-fold, respectively). The expression levels of five miRNAs (miR-130a, -148b, -29a, -29c, and -301a) were significantly downregulated in cervical cancer tissues compared with adjacent non-cancerous tissues. Notably, among 10 miRNAs, miRNA-130a and miRNA-148b exhibited lower expression levels (by 3-fold) in cervical cancers compared with normal cervical tissues. Synthetic mimics of miRNA-130a and miRNA-148b were transfected individually into HeLa cells. qRT-PCR and western blotting results showed that overexpression of miRNA-130a could effectively downregulate Dicer expression to a greater extent than miRNA-148b (see Supplementary Figure 1).

MiR-130a directly targets Dicer in cervical cancer cells. Out of the 10 miRNAs, miR-130a had the strongest relationship with Dicer and was predicted to target Dicer by most of the programs. This prompted us to focus on whether and how miR-130a targeted Dicer. To test the repressive potential of miRNA-130a on Dicer expression, a synthetic mimic of miRNA-130a was transfected into SiHa cells, and qRT-PCR and western blotting were used to monitor endogenous Dicer expression. Cells were also transfected with a scrambled mimic. The results showed that overexpression of 
Real-time PCR
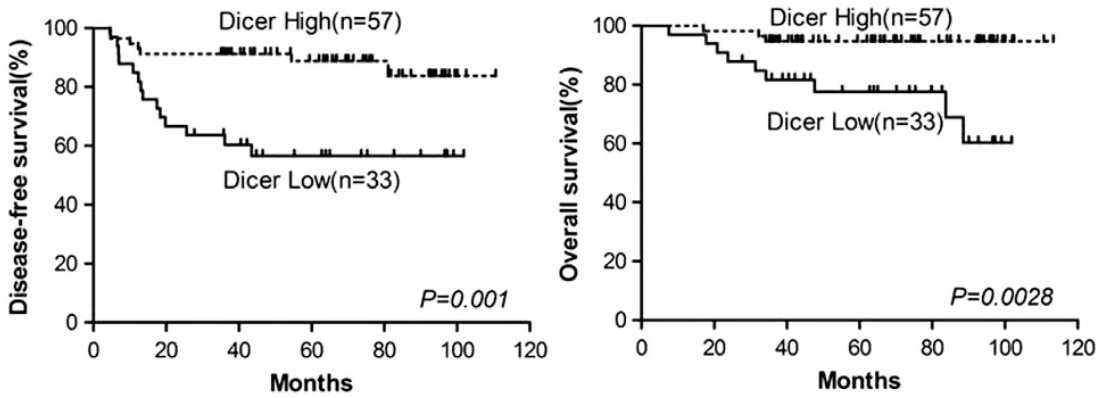

b

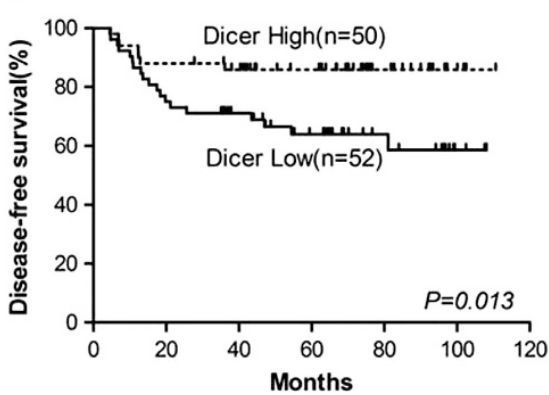

IHC

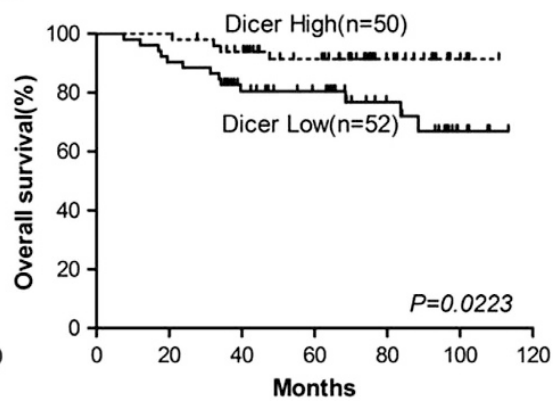

Figure 2 Cervical cancer patients with low Dicer expression had a significantly shorter disease-free survival and overall survival than those with high expression of Dicer at both the mRNA (a) and protein levels (b)

Table 2 Cox regression analysis of factors associated with disease-free survival and overall survival in cervical cancer patients

\begin{tabular}{|c|c|c|c|c|c|}
\hline \multirow[t]{2}{*}{ Variables } & \multirow[t]{2}{*}{ Favorable/Unfavorable } & \multicolumn{2}{|l|}{ IHC data sets } & \multicolumn{2}{|c|}{ Real-time PCR data sets } \\
\hline & & $\mathrm{HR}(95 \% \mathrm{Cl})$ & $\boldsymbol{P}$ & HR (95\% Cl) & $P$ \\
\hline \multicolumn{6}{|l|}{ Disease-free survival } \\
\hline $\begin{array}{l}\text { Age } \\
\text { SCC } \\
\text { Tumor size } \\
\text { Histology } \\
\text { FIGO Stage } \\
\text { Tumor grade } \\
\text { Lymph node metastasis } \\
\text { Dicer }\end{array}$ & $\begin{array}{l}>35 / \leqslant 35 \\
<1.5 \mathrm{ng} / \mathrm{ml} / \geqslant 1.5 \mathrm{ng} / \mathrm{ml} \\
<4 \mathrm{~cm} / \geqslant 4 \mathrm{~cm} \\
\text { Squamous/adenocarcinoma } \\
\text { I, II/III ,IV } \\
\text { G1/G2 or G3 } \\
\text { No/yes } \\
\text { High/low }\end{array}$ & $\begin{array}{l}1.157(0.464-2.885) \\
1.181(0.513-2.718) \\
0.955(0.443-2.061) \\
1.377(0.324-5.84) \\
3.662(1.582-8.475) \\
1.296(0.305-5.506) \\
1.588(0.64-3.939) \\
2.859(1.201-6.804)\end{array}$ & $\begin{array}{l}0.754 \\
0.695 \\
0.907 \\
0.665 \\
0.002 \\
0.755 \\
0.319 \\
0.018\end{array}$ & $\begin{array}{l}1.322(0.512-3.413) \\
1.171(0.454-3.02) \\
1.16(0.492-2.732) \\
1.985(0.458-8.607) \\
4.427(1.277-15.342) \\
0.998(0.402-2.477) \\
1.505(0.607-3.735) \\
4.105(1.652-10.197)\end{array}$ & $\begin{array}{l}0.564 \\
0.744 \\
0.599 \\
0.177 \\
0.019 \\
0.36 \\
0.996 \\
0.002\end{array}$ \\
\hline $\begin{array}{l}\text { Multivariate analysis } \\
\text { FIGO Stage } \\
\text { Dicer }\end{array}$ & $\begin{array}{l}\text { I, II/III, IV } \\
\text { High/low }\end{array}$ & $\begin{array}{l}5.595(1.302-14.036) \\
2.969(1.122-7.859)\end{array}$ & $\begin{array}{l}0.021 \\
0.072\end{array}$ & $\begin{array}{l}2.844(0.8-10.106) \\
3.715(1.467-9.403)\end{array}$ & $\begin{array}{l}0.106 \\
0.006\end{array}$ \\
\hline \multicolumn{6}{|l|}{$\begin{array}{l}\text { Overall survival } \\
\text { Univariables }\end{array}$} \\
\hline $\begin{array}{l}\text { Age } \\
\text { SCC } \\
\text { Tumor size } \\
\text { Histology } \\
\text { FIGO Stage } \\
\text { Tumor grade } \\
\text { Lymph node metastasis } \\
\text { Dicer }\end{array}$ & $\begin{array}{l}>35 / \leqslant 35 \\
<1.5 \mathrm{ng} / \mathrm{ml} / \geqslant 1.5 \mathrm{ng} / \mathrm{ml} \\
<4 \mathrm{~cm} / \geqslant 4 \mathrm{~cm} \\
\text { Squamous/adenocarcinoma } \\
\text { I, II/III, IV } \\
\text { G1/G2 or G3 } \\
\text { No/yes } \\
\text { High/low }\end{array}$ & $\begin{array}{l}1.046(0.34-3.218) \\
2.126(0.808-5.596) \\
1.474(0.561-3.874) \\
2.472(0.562-10.872) \\
6.794(2.548-18.117) \\
2.004(0.263-15.245) \\
2.279(0.721-7.208) \\
3.418(1.114-10.489)\end{array}$ & $\begin{array}{l}0.937 \\
0.127 \\
0.432 \\
0.231 \\
0 \\
0.37 \\
0.161 \\
0.032\end{array}$ & $\begin{array}{l}1.461(0.438-4.872) \\
1.641(0.493-5.457) \\
2.286(0.688-7.596) \\
4.736(0.996-22.515) \\
7.761(1.626-37.046) \\
1.486(0.47-4.7) \\
2.152(0.68-6.814) \\
5.794(1.568-21.415)\end{array}$ & $\begin{array}{l}0.538 \\
0.419 \\
0.893 \\
0.177 \\
0.01 \\
0.051 \\
0.501 \\
0.008\end{array}$ \\
\hline $\begin{array}{l}\text { Multivariate analysis } \\
\text { FIGO Stage } \\
\text { Dicer }\end{array}$ & $\begin{array}{l}\text { I, II/III, IV } \\
\text { High/low }\end{array}$ & $\begin{array}{c}10.742(1.638-23.726) \\
2.346(0.757-7.272)\end{array}$ & $\begin{array}{l}0.002 \\
0.152\end{array}$ & $\begin{array}{l}4.982(1.019-24.371) \\
5.13(1.363-19.307)\end{array}$ & $\begin{array}{l}0.047 \\
0.016\end{array}$ \\
\hline
\end{tabular}

Abbreviations: $\mathrm{Cl}$, confidence interval; $\mathrm{HR}$, hazard ratio

miRNA-130a could effectively downregulate Dicer expression compared with the scrambled mimic. This indicated that miR-130a could inhibit Dicer expression.
To test whether miR-130a targeted Dicer directly, a luciferase reporter assay was performed. A dual-luciferase reporter vector containing the wild-type (Figure 4a) 

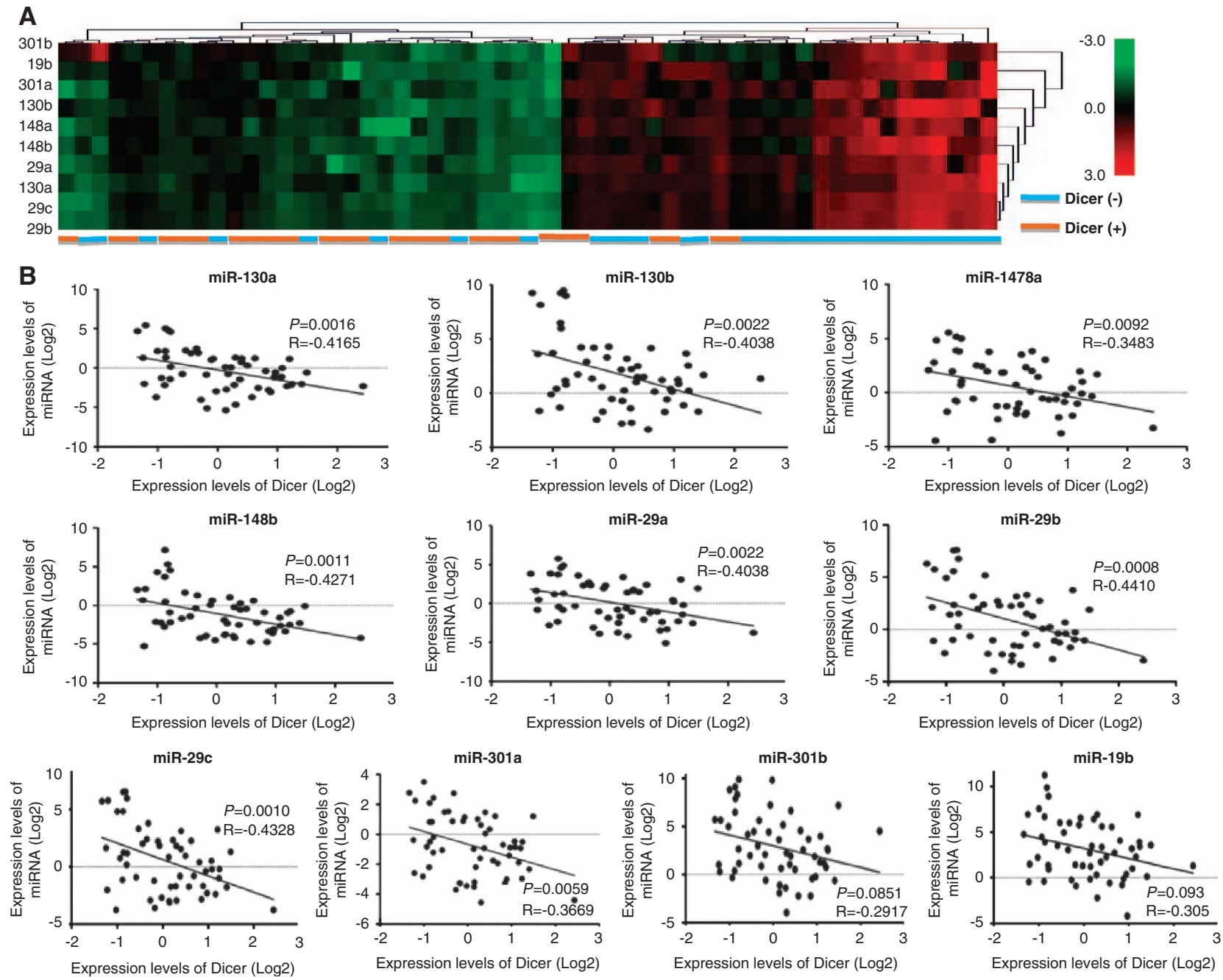

Figure 3 (a) Clustering analysis showing that the mRNA level of microRNAs in cervical cancer was negatively correlated with the Dicer mRNA expression level. (b) Correlation between Dicer and miRNA expressions in cervical cancer

Dicer 3'-UTR was co-transfected into 293T cell with miR-130a mimics, scrabbled sequences (as a negative control, NC) or transfection reagents (as a blank control, $\mathrm{BC}$ ). As expected, relative luciferase activity in 293T cells transfected with miR-130a mimics was significantly lower than that in the cells with scrabbled sequences or reagents (Figure 4b). To confirm that the reduced luciferase activity was specifically caused by miR-130a binding to the seed site of $3^{\prime}-U T R$, the seed sequence Dicer $3^{\prime}$-UTR was mutated in the luciferase reporter construct (Figure 4a). The result showed that luciferase activity in the cells containing the mutant reporter construct was the same when treated with miR-130a mimics, scrabbled sequences or transfection reagents. These results demonstrated that miR-130a could bind directly to Dicer $3^{\prime}$-UTR (Figure 4b).

We then asked whether miR-130a could suppress Dicer mRNA expression by binding to $3^{\prime}$-UTR of Dicer. Previous studies showed that let- $7 \mathrm{a}^{16,20}$ and $\mathrm{miR}-107^{21}$ also targeted Dicer. Therefore, to compare the inhibitory effect of miR-130a on Dicer expression with that of let-7a and miR-107, we transiently transfected $\mathrm{SiHa}$ cells with the three miRNA mimics, separately. To further verify that miR-130a targeted the Dicer $3^{\prime}$-UTR specifically, we also transfected miR-130a mutants into $\mathrm{SiHa}$ cells. qRT-PCR analysis showed that compared with NC, Dicer mRNA levels were reduced significantly by miR-130a and miR-107 (both $P<0.05$ ) and marginally significantly by let-7a $(P=0.0622)$, but not by miR130a-MUT (Figure 4c). To further confirm that the downregulated Dicer mRNA was caused specifically by these miRNAs, antagomiRs against these three miRNAs were transfected into SiHa cells. As shown in Figure 4c, the Dicer mRNA expression level was significantly increased in the cells with antagomiR-130a or antagomiR-107 (both $P<0.05$ ) and increased marginally significantly in cells with antagomiR let-7a, compared with cells with NC. Simultaneously, we also measured Dicer protein expression in $\mathrm{SiHa}$ cells transfected with these miRNA mimics and antagomiRs using western blotting. As shown in Figure 4d, similar results to the qRT-PCR experiment were observed. Taken together, these results indicated that miR-130a could target Dicer mRNA and markedly inhibited Dicer expression, comparably with miR-107 and stronger than let-7a. 
A

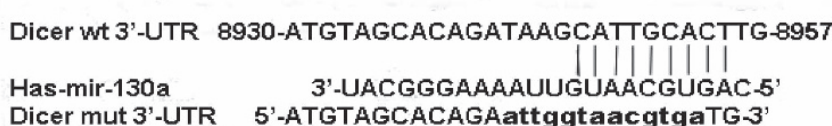

C

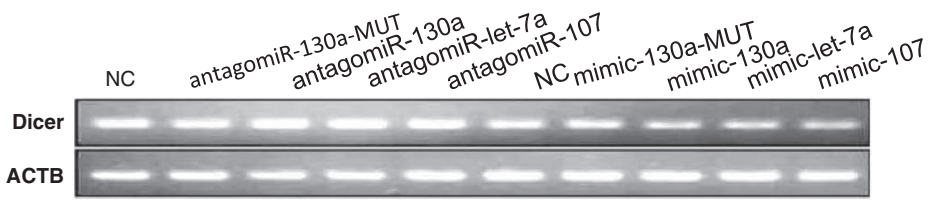

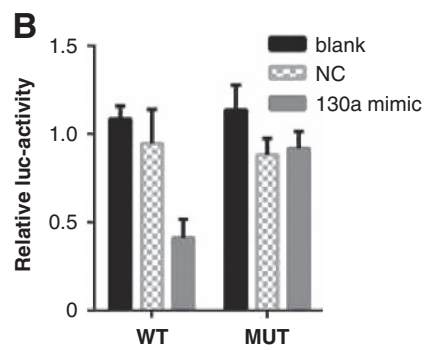
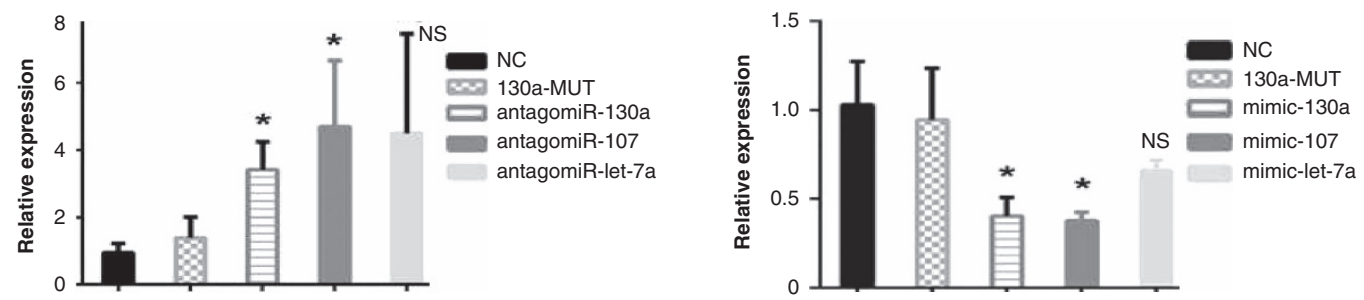

D
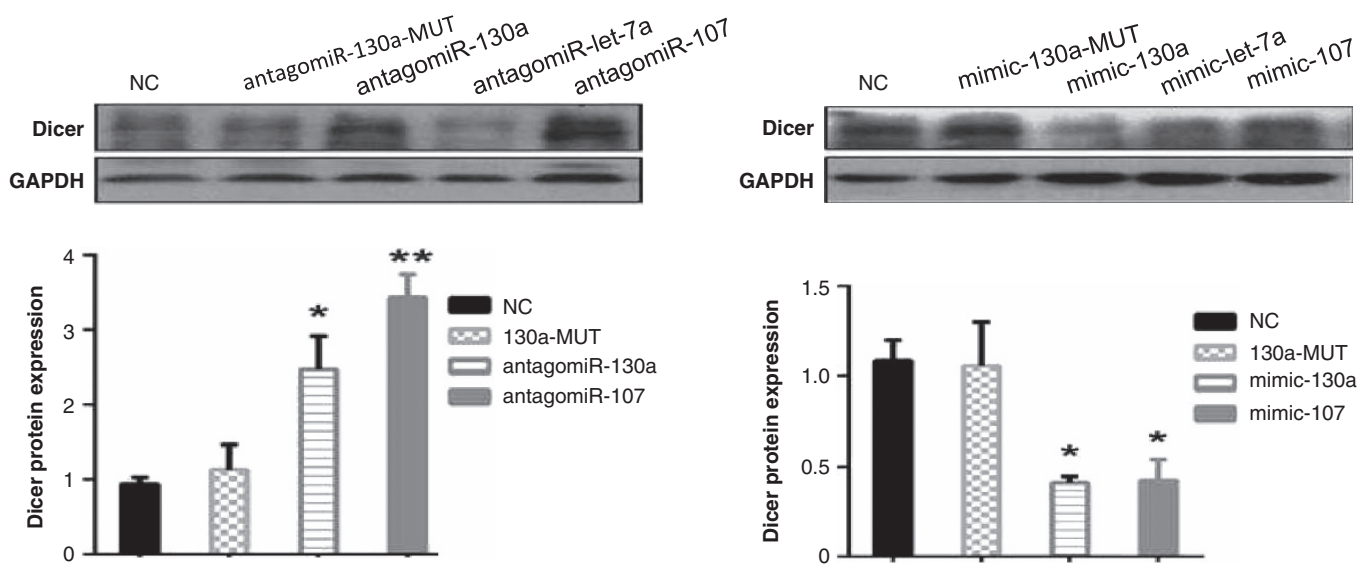

Figure 4 (a) Schematic illustration showing locations of the putative miR-130a target site and luciferase insert (Luc. insert) in the $3^{\prime}$-UTR of Dicer. The sequence alignment of mature miR-130a, its target site and mutated nucleotides are also shown. (b) Relative luciferase activity was measured using 293T cells and showed marked reduction following insertion of the $3^{\prime}$-UTR of wild-type (wt) Dicer, but not with the insertion of the $3^{\prime}$-UTR of the mutant (mut) Dicer. That reduction was significantly recovered following treatment with mimic-130a, but not with the negative control. (c) qRT-PCR analysis of expression level of dicer in SiHa cells transfected with antagomiRs and mimics. The mRNA level of Dicer in cervical cancer cell was upregulated by transfecting with antagomiR-130a and downregulated by transfecting with mimic-130a. (d) The protein level of Dicer was upregulated by antagomiRs (130a and 107) and downregulated by mimics (130a and 107); NC, negative control. We analyzed all versus control, except miR-130a, which we analyzed versus miR-130a-mut. ${ }^{*} P<0.05$ when compared with $\mathrm{NC}$; ${ }^{* *} P<0.01$ when compared with NC; NS means no significance when compared with NC.

\begin{abstract}
MiR-130a promotes migration and invasion of SiHa cells. To test our hypothesis that Dicer-targeted miRNAs might indirectly promote migration and invasion of cervical cancer cells, we employed a transwell assay to evaluate the effects of miR-130a expression on cell migration and invasion. Other Dicer-targeted miRNAs, let-7a and miR107, were also tested. As shown in Figure 5a, miR-130a, let-7a and miR-107 mimics noticeably increased SiHa cell migration, while antagomiRs against miR-130a, let-7a and miR-107 significantly decreased migration. Consistent results were observed in the cell invasion assay (Figure 5b). This in vitro evidence corroborated the observation that low Dicer expression correlated with metastasis of cervical cancer.
\end{abstract}

MiR-130a expression is associated with survival of patients with cervical cancer. Based on the above findings, we hypothesized that Dicer-targeted miRNAs miR-130a, let-7a and miR-107 might also be associated with survival. We detected the expression levels of miR-130a, let-7a and miR-107 by qRT-PCR in 73 cases out of 102 patients whose RNA was available. We then performed survival analysis in comparison with the expression levels of these miRNAs. The DFS of patients with low miRNA-130a or miR-107 expression was higher than that of patients with high miRNA-130a or miR-107 expression $(P=0.018$, $P=0.007$, respectively; Figures $6 a$ and $b$ ), and that of patients with low let-7a was marginally significantly higher than that of patients with high let-7a $(P=0.086$; Figure $6 \mathrm{c})$. 
a
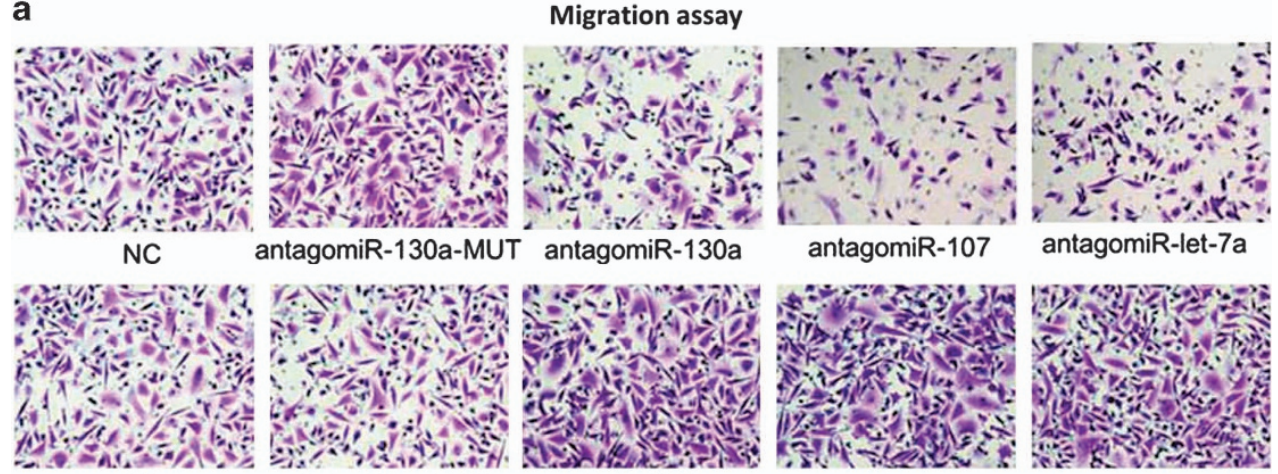

NC

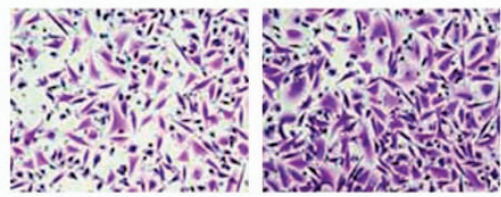

mimic-130a

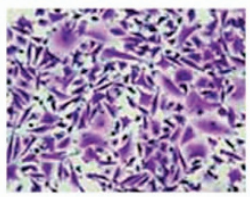

mimic-107

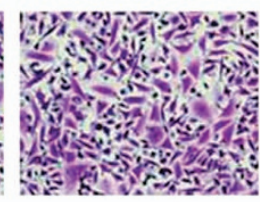

mimic-let-7a
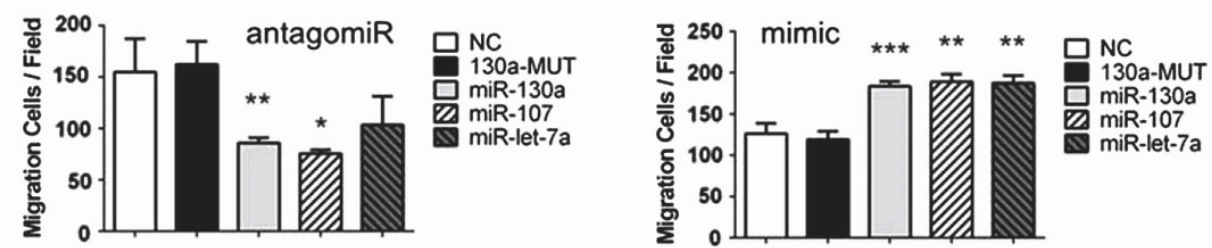

b

\section{Invasion assay}
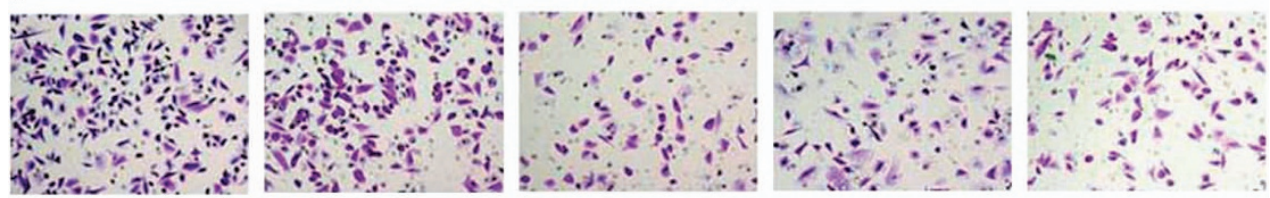

NC

antagomiR-130a-MUT antagomiR-130a

antagomiR-107

antagomiR-let-7a

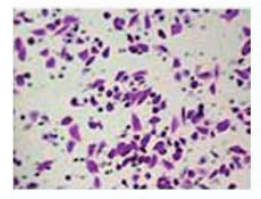

NC
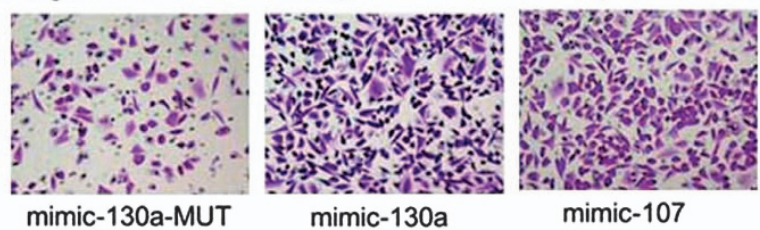

mimic-130a

mimic-107

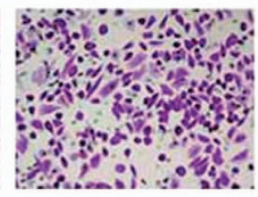

mimic-let-7a
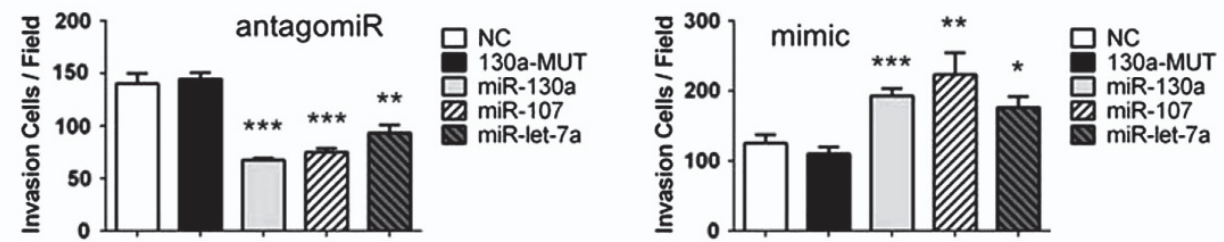

Figure 5 Transwell migration assays (a) and Matrigel invasion assays (b) of SiHa cells infected with antagomiRs, mimics and controls. Images shown at a magnification of $\times 200$. The $P$ values were calculated using Student's $t$-test. ${ }^{*} P<0.05$ versus control; ${ }^{* \star} P<0.01,{ }^{* \star} P<0.001$

The OS of patients with low miRNA expression (miR-130a, let-7a and miR-107) was not significantly higher than that of patients with high miRNAs $(P=0.172, P=0.175, P=0.101$, respectively; Figures $6 a-c)$. These clinical results were in agreement with biological functions of these miRNAs.

\section{Discussion}

In this study, we found that low expression of Dicer mRNA and protein correlated with poor prognosis and relapse (including distance metastasis) of cervical cancer. Low Dicer expression was associated with patients with metastatic relapse. Low protein expression of Dicer was significantly associated with tumor stage. Patients with low Dicer mRNA and protein expression showed a shorter 5-year DFS and OS. Thus, low expression of
Dicer seemed to be a significant prognostic factor for cervical cancer. These findings in cervical cancer are consistent with the results reported in the literature in other tumors. ${ }^{5,8}$

Although Dicer mRNA and protein expression levels in cervical cancer were much lower than those in normal cervical tissue, the reduced Dicer mRNA in cervical cancer was not significant compared with normal cervical tissue. The lower expression level of the Dicer protein compared with that of Dicer mRNA in cervical cancer might be mediated by protein regulation; for example, reduced levels of the TRBP protein, an integral component of the DICER1containing complex, resulted in a destabilization of the DICER1 protein. $^{22}$

The literature suggests possible mechanisms underlying Dicer mRNA downregulation in cancer. The Dicer gene locus 

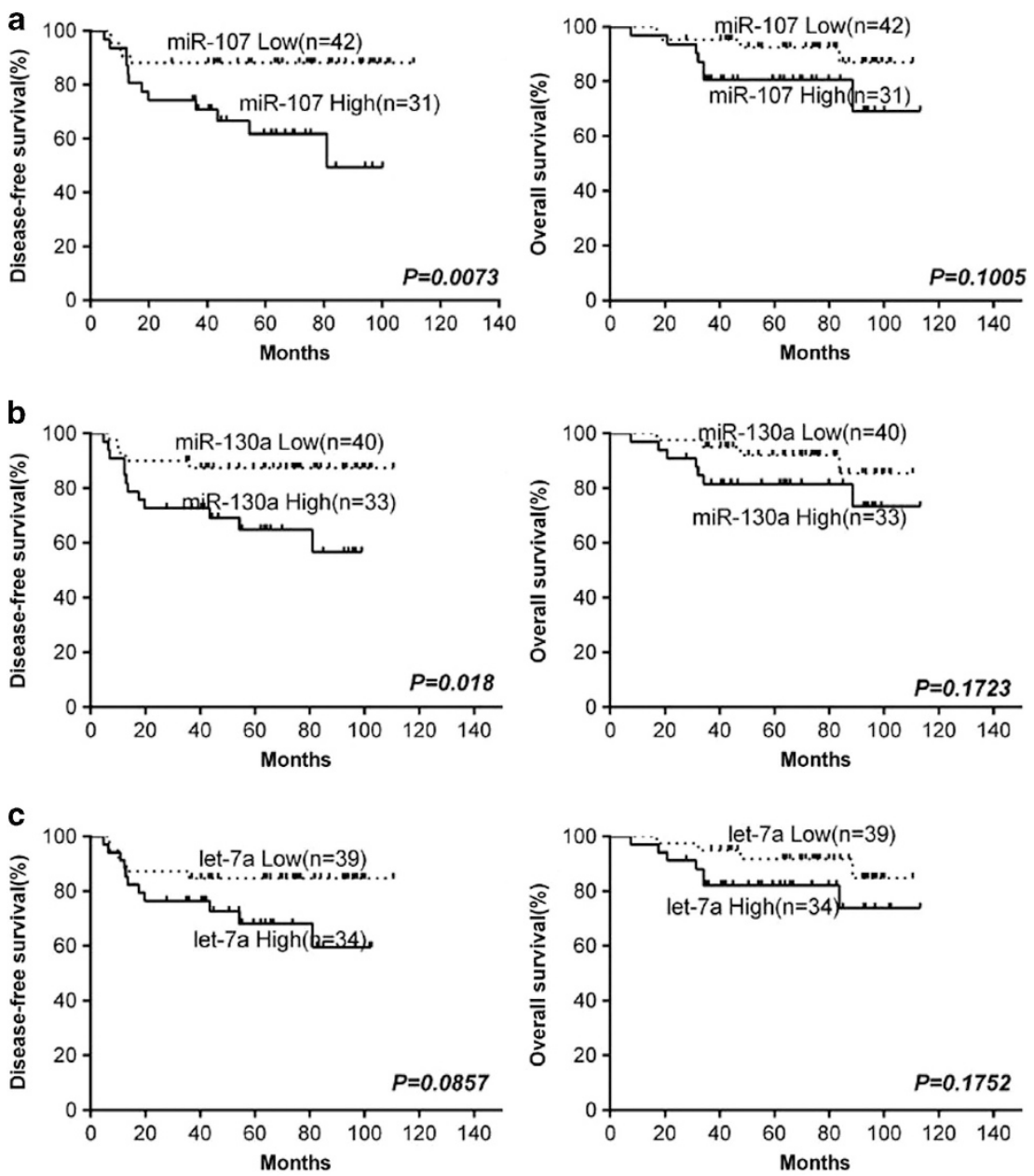

Figure 6 Kaplan-Meier graph representing the probability of disease-free survival and overall survival in cervical cancer patients $(n=73)$ according to the miR-107 (a), miR-130a (b) and let-7a (c) relative expression. The log-rank test $P$ value reflects the significance of the association between high miR-130a levels and metastasis

(on chromosome 14q) deletion might be one of the mechanisms in some cancers. ${ }^{23,24}$ In addition, higher expression of miRNAs that target Dicer might be another critical mechanism, for example, miR103/107 were reported to target and downregulate Dicer expression and be associated with poor survival in breast cancer ${ }^{15}$ and gastric cancer. ${ }^{25}$ let-7 expression was inversely correlated with Dicer expression and constituted a negative feedback loop controlling Dicer expression in a panel of cancer cell lines. ${ }^{16}$ Interestingly, miR-107 can directly interact with let-7 and reduce its expression, ${ }^{26}$ suggesting a complex relationship between miR-107, let-7 and Dicer. More important, recent evidence shows that aberrant miRNA expression has important roles in cervical cancer. ${ }^{27,28}$ These reports convinced us that miRNAs were involved in the low Dicer expression in cervical cancer. Therefore, we first identified 10 miRNAs that were predicted to target the $3^{\prime}$-UTR of Dicer transcript using several miRNA target prediction programs. qRT-PCR showed that low Dicer expression was correlated significantly and inversely with overexpression of the 10 miRNAs in cervical cancer. We then focused on miR130a. Luciferase reporter assays with wild-type or mutated Dicer 3'-UTR, and cervical cancer cell gain- or loss-of-function assay with miR-130a mimics, mutants or antagomiRs indicated that miR-130a targeted Dicer directly.

In our study, miR-130a functions as an oncogene in cervical cancer, which is consistent with the data reported by Liu et al. in colon cancer ${ }^{29}$ and Wang et al. in NSCLC. ${ }^{14}$ However, some reports showed that miR-130a functioned as a tumor suppressor ${ }^{30-32}$ The reasons for the apparently contradictory roles of miR-130a are not clear. One reason is that miR-130a may have different roles in different circumstances or tissues.

Recently, Su et al. ${ }^{33}$ found that tumor suppressor Tap63 could activate Dicer and miR-130b (one of the miR-130 family) at the promoter level to inhibit tumor metastasis. In contrast with the tumor suppressor role of miR-130b in Su's report, miR-130a was observed to have an oncogenic role in our study (i.e., it promotes cancer cell migration and invasion), suggesting that they might target different genes under different conditions. Surprisingly, however, we found that six miRNA-target prediction programs predicted Tap63 as a target of miR-130a and miR-130b, implying that miRNAs, Dicer and Tap63 might constitute a complex and accurate regulation circuit playing an important role in cancer development and progression. 
In conclusion, we report, for the first time, that Dicer expression is an important prognostic factor in cervical cancer. Low Dicer expression is associated with decreased 5 -year OS and DFS. Moreover, we present evidence that several miRNAs may affect Dicer expression. High expression of miRNA-130a attenuated Dicer expression. These results further increase our knowledge concerning the mechanisms of RNA interference. Finally, our findings may identify novel targets for the treatment of cervical cancer.

\section{Materials and Methods}

Cervical cancer samples and cell lines. This study was approved by the Review Board of our Cancer Center of Sun Yat-sen University. Formalin-fixed tissues were obtained from patients with invasive cervical cancer who underwent surgical resection at the Sun Yat-Sen University Cancer Center, Guangzhou, China, between 2002 and 2008. Ninety cancer tissues and 23 paired adjacent nontumorous tissues were used for the GRT-PCR study. None of the patients had received chemotherapy or radiotherapy before surgery. One hundred and two paraffin-embedded tissues were used for immunohistochemistry, which included the above-mentioned 90 cancer tissues and another 12 patients who underwent cervical biopsy and accepted chemotherapy or radiotherapy. Thirty-four patients had paired adjacent non-tumorous tissues among the 102 paraffin-embedded tissues. Two pathologists diagnosed all the samples. The follow-up data in this study were available and complete. Histological types were assigned according to the WHO classification criteria. The 5 -year DFS was $52.9 \%$, with a median follow-up time of 5.27 years; the 5 -year OS was $61.8 \%$, with a median follow-up time of 5.70 years.

Cell lines. The cervical cancer cell lines HeLa and SiHa were cultured in RPMI 1640 supplemented with $10 \%$ heat-inactivated fetal bovine serum (FBS) and $1 \%$ penicillin-streptomycin. All cells were incubated at $37^{\circ} \mathrm{C}$ in a humidified chamber containing $5 \% \mathrm{CO}_{2}$

RNA extraction and real-time PCR. Total RNA from cultured cells and fresh frozen tissues was extracted using Trizol reagent (Invitrogen, Carlsbad, CA, USA), according to the manufacturer's instructions. Reverse transcriptase reactions using MMLV reverse transcriptase reagents (Promega, Madison, WI, USA) were performed following the manufacturer's protocol. PCR conditions were $95^{\circ} \mathrm{C}$ for $10 \mathrm{~min}$; followed by $45 \mathrm{cycles}$ of $95^{\circ} \mathrm{C}$ for $30 \mathrm{~s}$ and $60^{\circ} \mathrm{C}$ for $1 \mathrm{~min}$ The primer pairs used for Dicer were $5^{\prime}$-ACACCTTTACCTGATGAACT- $3^{\prime}$ and $5^{\prime}$-GTGTGGAATCTGAGGTATGG-3' , and for ACTB were $5^{\prime}$-ATGTGGCCGAGGA CTTTGATT-3' and 5'-AGTGGGGTGGCTTTTAGGATG-3'. For miRNA detection, reverse transcription followed by stem-loop qRT-PCR was performed according to the manufacturer's protocols, using the Bulge-LoopTM miRNA qRT-PCR Primer (RiboBio, Guangzhou, China). Real-time PCR was performed using Platinum SYBR Green qPCR SuperMix-UDG reagents (Invitrogen) in an Applied Biosystems PRISM $7900 \mathrm{HT}$ instrument. Expression variations were calculated using the $2^{-\Delta \Delta \mathrm{Ct}}$ method. Total RNA input was normalized based on threshold cycle (Ct) values of common internal control for miRNA quantification assays, U6 snRNA, and all Ct values $\geq 36$, which were considered as not expressed, were adjusted to 36

Immunohistochemistry. Immunohistochemistry was performed according to standard methods, as previously described. ${ }^{34}$ The anti-Dicer antibody used for the staining was a mouse monoclonal antibody (at a dilution of 1:400) (Abcam, Cambridge, UK). Dicer staining was observed in the cytoplasm. Control samples were stained in parallel, but were not incubated with either primary or secondary antibodies. The intensity of staining was graded on a scale from 0 to 3 . Slides given a score of 0 represented no immunoreactivity, and a score of 3 represented strong immunostaining. Two pathologists confirmed the results in a double-blind analysis. Scores $<2$ were recorded as negative expression, while scores $\geq 2$ were recorded as positive expression.

MiRNA target prediction. In silico prediction of miRNAs that might target Dicer was performed using the algorithms PicTar (http://pictar.mdc-berlin.de/), ${ }^{35}$ TargetScan (http://www.targetscan.org/) ${ }^{36}$ and miRanda (http://www.microrna.org/ microrna/); ${ }^{37}$ Venn diagram analysis was then performed to identify miRNAs that were conserved in three species.
Transfection. MiRNA mimics were transfected into cells at a final concentration of $50 \mathrm{nmol} / \mathrm{l}$ using Lipofectamine RNAi MAX reagent (Invitrogen). Cells were incubated with miRNA mimics and appropriate scramble controls (all from GenePharma Company, Shanghai, China) for $4 \mathrm{~h}$ in Opti-MEM media before the addition of normal growth medium. The cells were then assayed for $48 \mathrm{~h}$ after transfection.

Western blot analysis. Western blotting was performed according to standard methods (for details, see Supplementary Methods), using an anti-DICER antibody (1:400; Abcam, Cambridge, UK) as the primary antibody.

Luciferase reporter assay. Briefly, 50000 cells were seeded in one well of a six-well plate, in triplicate, and allowed to settle for $12 \mathrm{~h}$. $293 \mathrm{~T}$ cells were transfected with $100 \mathrm{ng}$ E-box reporter-luciferase plasmid or $100 \mathrm{ng}$ controlluciferase plasmid plus $10 \mathrm{ng} \mathrm{pRL}-\mathrm{TK}$ renilla plasmid using the Lipofectamine 2000 reagent (Invitrogen). Media were replaced at $6 \mathrm{~h}$, and the luciferase and the renilla signals were measured $48 \mathrm{~h}$ after transfection using the Dual Luciferase Reporter Assay Kit (Promega Corporation, Beijing, China), according to the manufacturer's protocol.

Cell migration assay and invasion assay. For the transwell migration assay, $2 \times 10^{5}$ cells were placed in the top chamber of each insert (BD, Durham, NC, USA), without matrigel coating. For the invasion assay, $2 \times 10^{5}$ cells were placed on the upper chamber of each insert, which was coated with $0.5 \mathrm{mg} / \mathrm{ml}$ Matrix gel Basement Membrane Matrix (BD) (for details, see Supplementary Methods).

Statistical analysis. The best cutoff value for separating two groups in terms of gene expression levels (log2 of Dicer) was determined by Student's $t$-test. The association between various clinical characteristics and expression levels of Dicer was examined by the chi-square test or by Fisher's exact test. DFS and OS were estimated with the Kaplan-Meier method, and were compared by log-rank test using GraphPad Prism software (version5, GraphPad Software, La Jolla, CA, USA). Cox regression analysis was used to assess factors related to survival. All statistical analyses were performed with SPSS 16.0 software (SPSS Inc, Chicago, IL, USA). A $P$ value $<0.05$ was considered significant.

\section{Conflict of Interest}

The authors declare no conflict of interest.

Acknowledgements. This work was supported by grants from the National Natural Science Foundation of China (grant numbers 81171948, 81372275), the Key Program of Natural Science Foundation of Guangdong Province, China (grant number S2012020011060) and the Project of State Key Laboratory of Oncology in South China (grant number 030041060004).

1. Kuehbacher A, Urbich C, Zeiher AM, Dimmeler S. Role of Dicer and Drosha for endothelial microRNA expression and angiogenesis. Circ Res 2007; 101: 59-68.

2. Tang KF, Wang Y, Wang P, Chen M, Chen Y, Hu HD et al. Upregulation of PHLDA2 in Dicer knockdown HEK293 cells. Biochim Biophys Acta 2007; 1770: 820.

3. Barbato C, Ciotti MT, Serafino A, Calissano P, Cogoni C. Dicer expression and localization in post-mitotic neurons. Brain Res 2007; 1175: 17-27.

4. Bernstein E, Kim SY, Carmell MA, Murchison EP, Alcorn H, Li MZ et al. Dicer is essential for mouse development. Nat Genet 2003; 35: 215-217.

5. Karube $\mathrm{Y}$, Tanaka H, Osada H, Tomida S, Tatematsu Y, Yanagisawa $\mathrm{K}$ et al. Reduced expression of Dicer associated with poor prognosis in lung cancer patients. Cancer Sci 2005; 96: 111-115

6. Grelier G, Voirin N, Ay AS, Cox DG, Chabaud S, Treilleux I et al. Prognostic value of Dicer expression in human breast cancers and association with the mesenchymal phenotype. Br J Cancer 2009; 101: 673-683

7. Zighelboim I, Reinhart AJ, Gao F, Schmidt AP, Mutch DG, Thaker PH et al. DICER1 expression and outcomes in endometrioid endometrial adenocarcinoma. Cancer 2011; 117: $1446-1453$

8. Merritt WM, Lin YG, Han LY, Kamat AA, Spannuth WA, Schmandt R et al. Dicer, Drosha, and outcomes in patients with ovarian cancer. N Engl J Med 2008; 359: 2641-2650.

9. Behm-Ansmant I, Rehwinkel J, Izaurralde E. MicroRNAs silence gene expression by repressing protein expression and/or by promoting mRNA decay. Cold Spring Harb Symp Quant Biol 2006; 71: 523-530. 
10. Friedman RC, Farh KK, Burge CB, Bartel DP. Most mammalian mRNAs are conserved targets of microRNAs. Genome Res 2009; 19: 92-105.

11. Jannot G, Simard MJ. Tumour-related microRNAs functions in Caenorhabditis elegans. Oncogene 2006; 25: 6197-6201.

12. Vandenboom li TG, Li Y, Philip PA, Sarkar FH. MicroRNA and cancer: tiny molecules with major implications. Curr Genomics 2008; 9: 97-109.

13. Chen Y, Gorski DH. Regulation of angiogenesis through a microRNA (miR-130a) that down-regulates antiangiogenic homeobox genes GAX and HOXA5. Blood 2008; 111: 1217-1226.

14. Wang $X C$, Tian $L L$, Wu HL, Jiang $X Y$, Du LQ, Zhang $H$ et al. Expression of miRNA-130a in nonsmall cell lung cancer. Am J Med Sci 2010; 340: 385-388.

15. Martello G, Rosato A, Ferrari F, Manfrin A, Cordenonsi M, Dupont S et al. A MicroRNA targeting dicer for metastasis control. Cell 2010; 141: 1195-1207.

16. Tokumaru S, Suzuki M, Yamada H, Nagino M, Takahashi T. let-7 regulates Dicer expression and constitutes a negative feedback loop. Carcinogenesis 2008; 29: 2073-2077.

17. Lui WO, Pourmand N, Patterson BK, Fire A. Patterns of known and novel small RNAs in human cervical cancer. Cancer Res 2007; 67: 6031-6043.

18. Wang X, Tang S, Le SY, Lu R, Rader JS, Meyers C et al. Aberrant expression of oncogenic and tumor-suppressive microRNAs in cervical cancer is required for cancer cell growth. PLoS One 2008; 3: e2557.

19. Huang L, Lin J-X, Yu Y-H, Zhang M-Y, Wang H-Y, Zheng $M$ et al. Down regulation of six microRNAs is associated with advanced stage, lymph node metastasis and poor prognosis in small cell carcinoma of the cervix. PLoS One 2012; 7: e33762.

20. Forman JJ, Legesse-Miller A, Coller HA. A search for conserved sequences in coding regions reveals that the let-7 microRNA targets Dicer within its coding sequence. Proc Natl Acad Sci USA 2008; 105: 14879-14884.

21. Li X, Zhang Y, Shi Y, Dong G, Liang J, Han Y et al. MicroRNA-107, an oncogene microRNA that regulates tumour invasion and metastasis by targeting DICER1 in gastric cancer. J Cell Mol Med 2011; 15: 1887-1895.

22. Melo SA, Ropero S, Moutinho C, Aaltonen LA, Yamamoto H, Calin GA et al. A TARBP2 mutation in human cancer impairs microRNA processing and DICER1 function. Nat Genet 2009; 41: 365-370.

23. Thompson PM, Seifried BA, Kyemba SK, Jensen SJ, Guo C, Maris JM et al. Loss of heterozygosity for chromosome 14q in neuroblastoma. Med Pediatr Oncol 2001; 36: 28-31.

24. Buffart TE, Carvalho B, van Grieken NC, van Wieringen WN, Tijssen M, Kranenbarg EM et al. Losses of chromosome $5 q$ and $14 q$ are associated with favorable clinical outcome of patients with gastric cancer. Oncologist 2012; 17: 653-662.

25. Inoue $T$, linuma $H$, Ogawa $E$, Inaba $T$, Fukushima R. Clinicopathological and prognostic significance of microRNA-107 and its relationship to DICER1 mRNA expression in gastric cancer. Oncol Rep 2012; 27: 1759-1764.

26. Chen PS, Su JL, Cha ST, Tarn WY, Wang MY, Hsu HC et al. miR-107 promotes tumor progression by targeting the let-7 microRNA in mice and humans. J Clin Invest 2011; 121: 3442-3455.
27. Shen SN, Wang LF, Jia YF, Hao YQ, Zhang L, Wang H. Upregulation of microRNA-224 is associated with aggressive progression and poor prognosis in human cervical cancer. Diagn Pathol 2013; 8: 69.

28. Luo M, Shen D, Zhou X, Chen X, Wang W. MicroRNA-497 is a potential prognostic marker in human cervical cancer and functions as a tumor suppressor by targeting the insulin-like growth factor 1 receptor. Surgery 2013; 153: 836-847.

29. Liu L, Nie J, Chen L, Dong G, Du X, Wu X et al. The oncogenic role of microRNA-130a/ $301 \mathrm{a} / 454$ in human colorectal cancer via targeting Smad4 expression. PLOS One 2013 8: e55532.

30. Qiu S, Lin S, Hu D, Feng Y, Tan Y, Peng Y. Interactions of miR-323/miR-326/miR-329 and $\mathrm{miR}-130 \mathrm{a} / \mathrm{miR}-155 / \mathrm{miR}-210$ as prognostic indicators for clinical outcome of glioblastoma patients. J Transl Med 2013; 11: 10.

31. Boll K, Reiche K, Kasack K, Morbt N, Kretzschmar AK, Tomm JM et al. MiR-130a, miR-203 and miR-205 jointly repress key oncogenic pathways and are downregulated in prostate carcinoma. Oncogene 2013; 32: 277-285.

32. Kovaleva V, Mora R, Park YJ, Plass C, Chiramel Al, Bartenschlager R et al. miRNA-130a targets ATG2B and DICER1 to inhibit autophagy and trigger killing of chronic lymphocytic leukemia cells. Cancer Res 2012; 72: 1763-1772.

33. Su X, Chakravarti D, Cho MS, Liu L, Gi YJ, Lin YL et al. TAp63 suppresses metastasis through coordinate regulation of Dicer and miRNAs. Nature 2010; 467: 986-990.

34. He L, Ding H, Wang JH, Zhou Y, Li L, Yu YH et al. Overexpression of karyopherin 2 in human ovarian malignant germ cell tumor correlates with poor prognosis. PLoS One 2012; 7: e42992.

35. Krek A, Grun D, Poy MN, Wolf R, Rosenberg L, Epstein EJ et al. Combinatorial microRNA target predictions. Nature Genet 2005; 37: 495-500.

36. Lewis BP, Burge CB, Bartel DP. Conserved seed pairing, often flanked by adenosines, indicates that thousands of human genes are microRNA targets. Cell 2005; 120: 15-20.

37. Betel D, Wilson M, Gabow A, Marks DS, Sander C. The microRNA.org resource: targets and expression. Nucleic acids research 2008Nucleic Acids Res 36: D149-D153.

Cell Death and Disease is an open-access journal published by Nature Publishing Group. This work is licensed under a Creative Commons Attribution 3.0 Unported License. The images or other third party material in this article are included in the article's Creative Commons license, unless indicated otherwise in the credit line; if the material is not included under the Creative Commons license, users will need to obtain permission from the license holder to reproduce the material. To view a copy of this license, visit http://creativecommons.org/licenses/by/3.0/

Supplementary Information accompanies this paper on Cell Death and Disease website (http://www.nature.com/cddis) 